\title{
SIGNATURES OF THE QUARK GLUON PLASMA: A PERSONAL OVERVIEW
}

\author{
C. GREINER \\ Institut für Theoretische Physik, Universität Giessen, D-35392 Giessen, Germany \\ E-mail: carsten.greiner@theo.physik.uni-giessen.de
}

\begin{abstract}
In this talk 'Signatures of the Quark-Gluon Plasma' are being reviewed. We first discuss, on a no-QGP basis, the two prominent indications of (a) enhanced strangeness production and of (b) anomalous $J / \psi$-suppression: We elaborate in particular on a recent idea of antihyperon production solely by multi-mesonic reactions. As a possible source for an enhanced dissociation of $c \bar{c}$ pairs we summarize the findings within the 'early'-comover absorption scenario of prehadronic string excitations. As an exotic candidate we then finally adress the stochastic formation of so called disoriented chiral condensates: An experimentally feasible DCC, if it does exist, has to be a rare event following an unusual and nontrivial distribution on an event by event basis.
\end{abstract}

\section{Motivation and Summary}

The prime intention for present ultrarelativistic heavy ion collisions at CERN and at Brookhaven lies in the possible experimental identification of the quark gluon plasma (QGP), a theoretically hypothesized new phase of matter, where quarks and gluons are deliberated and move freely over an extended, macroscopically large region. Recently, refering to several different experimental findings within the Lead Beam Programme at the CERN-SPS, strong 'circumstantial evidence' for the temporal formation of the QGP has been conjectured I. As a first and principle objection, however, any theoretical predictions in favor for the QGP can, strictly speaking, only be regarded as qualitative or as semi-quantitative: A satisfactory theoretical understanding of either the microscopic dynamics or of the hadronisation of a hypothetical deconfined phase is at present not really given. It is also of scientific importance still to confront the excitement with further possible criticism. We therefore want to review, on a no-QGP basis, where quantitaive predictions are possible, the twg most prominent indications for the QGP: enhanced strangeness production 3 and anomalous $J / \psi$-suppression 3 , both being proposed already a long time ago.

The main idea behind the collective enhancement of strangeness is that the strange (and antistrange) quarks are thought to be produced more easily and hence also more abundantly in such a deconfined state as compared to the production via highly threshold suppressed inelastic hadronic collisions.

luedcg: submitted to World Scientific on October 25, 2018 
The analysis of measured abundancies of hadronic particles within thermal models $\$$ strongly supports the idea of having established an equilibrated fireball in some late stage of the reaction. In this respect, especially a nearly fully chemically equilibrated yield of strange antibaryons, the aptihyperons, had originally been advocated as the appropriate QGP candidatel. Although intriguing, after all this may not be the correct interpretation of the observed antihyperon yields: In the following section 2 we will elaborate in brief on our very recent, yet conservative idea $\bar{b}^{-}$of rapid antihyperon production solely by multi-mesonic reactions like $n_{1} \pi+n_{2} K \rightarrow \bar{Y}+p$. This might indeed well explain the observed excess of antihyperons.

A suppression of the $J / \Psi$ yield in ultra-relativistic heavy-ion collisions (in comparison to Drell-Yan pairs) is seen as the other plausible signature because the strongly bound $J / \Psi$ should dissolve in the QGP due to color screening. Indeed, a significant reduction of the $J / \Psi$ yield when going from proton-nucleus to nucleus-nucleus collisions has been observed, especially for $\mathrm{Pb}+\mathrm{Pb}$ at $160 \mathrm{GeV} / \mathrm{A}$. Besides the QGP as a possible explanation more conservative views of possible $J / \Psi$-absorption like on the still incoming nucleons and also on the produced secondaries, the 'comovers', have been envisaged with rather good success in explaining the data. In these hadronic scenarios, however, the to be assumed and unknown annihilation crosssections of the $c \bar{c}-$ states on the mesons are highly debated. As a further and intuitive appealing alternative we will consider in section 3 within a microscopic simulation the effect of $c \bar{c}$ dissociation on the individual, highly excited hadronic strings in the prehadronic phase of the heavy-ion collision. Such a picture can in fact be regarded as an 'early'-comover absorption scenariol. A satisfactorary agreement with the various data can be achieved by choosing one phenomenological parameter within a rather plausible range.

As a last and exotic candidate for a direct signature stemming from the QGP phase transition (and which can be adressed in RHIC experiments at Brookhaven) we then summarize our ideas of stochastic formation of so called disoriented chiral condensates (DCC). The idea of DCCU first appeared in a work of Anselm but it was made widely known due to Bjorken, and Rajagopal and Wilczek. The spontaneous growth and subsequent decay of these configurations emerging after a rapid chiral phase transition from the QGP to the hadronic world would give rise to large collective fluctuations in the number of produced low momentum neutral pions compared to charged pions. In section 4 we briefly summarize our recent findings on the important question on the the likeliness of an instability leading potentially to a large DCC yield of low momentum pions 8 . Our investigations show that an experimentally feasible DCC, if it does exist in nature, has to be a rare event with some finite prob- 


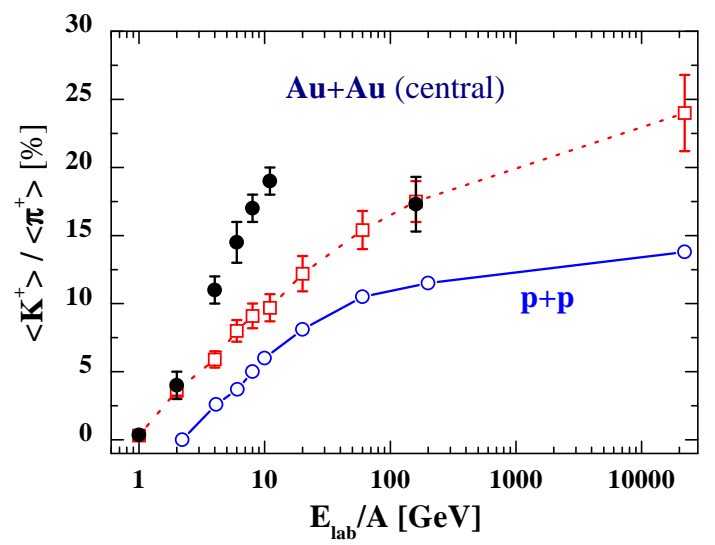

Figure 1. Calculated $K^{+} / \pi^{+}$-ratio 10 around midrapidity for central $\mathrm{Au}+\mathrm{Au}$ reactions (open squares) from SIS to RHIC energies in comparison to experimental data. For visualization of the collective strangeness enhancement the corresponding ratio for elementary $\mathrm{p}+\mathrm{p}$ collisions (open circles) is also depicted.

ability following a nontrivial and nonpoissonian distribution on an event by event basis. DCCs could then (only) be revealed experimentally by inspecting higher order factorial cumulants $\theta_{m}(m \geq 3)$ in the sampled distribution of low momentum pions.

\section{Strangeness and Antihyperon Production}

Since a relative enhancement of strangeness is observed already in hadronhadron collisions for increasing energy (see fig. 价, which is certainly not due to any macroscopic or bulk effects, the to be measured strangeness should be compared relative to $\mathrm{p}+\mathrm{p}$ collisions at the same energy. The arguments for enhanced strangeness production via the QGP should generally apply already for the most dominant strange particles, the kaonst. However, nonequilibrium inelastic hadronic reactions can explain to a very good extent the overall strangeness production seen experimentally 6 : Within a microscopic transport simulation an enhancement of the scaled kaon yield due to hadronic rescattering both with increasing system size and energy was found. The outcome for the most dominant strange particles, the $\mathrm{K}^{+}$-mesons, is summarized in 


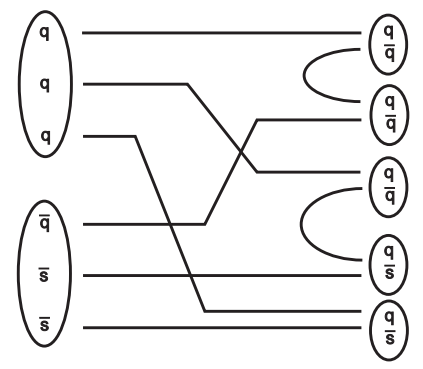

Figure 2. Schematic picture for $\bar{\Xi}+N \leftrightarrow 3 \pi+2 K$.

fig. 1. After the primary string fragmentation of intrinsic p-p-collisions the hadronic fireball starts with a $K^{+} / \pi^{+}$ratio still far below chemical equilibrium with $\approx 6-8 \%$ at AGS to SPS energies before the hadronic rescattering starts. The major amount of produced strange particles (kaons, antikaons and $\Lambda \mathrm{s}$ ) at CERN SPS-energies can then be understood in terms of early and still energetic, secondary and ternary non-equilibrium interactions. (At the lower AGS energies, the relative enhancement factor of $\approx 3$ can not be fully explained within the pure cascade type calculations 9 without any possible in-medium modifications of the kaons.)

Still, applying the usual concept of binary collisions within the transport approaches, the experimentally observed enhancement of antihyperons can by far not be explained by succesive binary (strangeness exchange) reactions This fact then gives the strong support for some new exotic mechanism like, most plausible, the temporary formation of a deconfined and strangeness saturated new state of matter $\boldsymbol{t}$. As outlined in the introduction, this might not be the full story 1 . Multimesonic 'back-reactions' (see eg fig. 2 for a particular illustration) involving $\mathrm{n}$ pions and $n_{Y}$ kaons of the type

$$
n \pi+n_{Y} K \leftrightarrow \bar{Y}+N
$$

corresponding to the inverse of the strong binary baryon-antibaryon annihilation process can easily account for a fast production of the antihyperon species. It is the latter annihilation process which dictates the timescale of how fast the antihyperon densities do approach local chemical equilibrium with the pions, nucleons and kaons. A simplified master equation for the number of antihyperons as a function of time can be written in the most direct form $\mathrm{F}$

$$
\frac{d}{d t} \rho_{\bar{Y}}=-\Gamma_{\bar{Y}}\left\{\rho_{\bar{Y}}-\rho_{\bar{Y}}^{e q}\right\},
$$


where production due to the multi-mesonic 'back-reactions' is hidden in the second term $\Gamma_{\bar{Y}} \rho_{\bar{Y}}^{e q}$. It is further plausible to assume that the annihilation crosssections are approximately the same like for $N \bar{p}$ at the same relative momenta, i.e. $\sigma_{p \bar{Y} \rightarrow n \pi+n_{Y} K} \approx 50 \mathrm{mb}$. The equilibration timescale $\left(\Gamma_{\bar{Y}}\right)^{(-1)} \sim$ $1 /\left(\sigma_{N \bar{Y}} v_{\bar{Y} N} \rho_{B}\right)$ is thus to a good approximation proportional to the inverse of the baryon density. Adopting an initial baryon density of approximately 1-2 times normal nuclear matter density $\rho_{0}$ for the initial and thermalized hadronic fireball, the antihyperons will equilibrate on a timescale of $1-3 \mathrm{fm} / \mathrm{c}$ ! This timescale competes with the expansion timescale of the late hadronic fireball, which is in the same range or larger. In any case it becomes clear that these multimesonic, hadronic reactions, contrary to binary reactions, can explain most conveniently a sufficiently fast equilibration before the (so called) chemical freeze-out occurs at the parameters given by the thermal model analysest.

To be more quantitative some explicite coupled master equations for an expanding system have to be considered and are presently pursued. In addition, one has to invent some clever strategy to handle such multi-particle 'back-reactions' within the sophisticated transport codes.

\section{$3 \quad J / \Psi$-suppression via Dissociation by Strings}

Within a microscopic hadronic transport calculation one can exploit various assumptions (models) for the $c \bar{c}$ formation and propagation and also take into account the Drell-Yan process explicitly. As one particular scenario we now report on the effect of $c \bar{c}$ dissociation in the prehadronic phase of the heavy-ion collisiond. This is motivated by the fact, that the very early collision phase is not described by hadrons but by highly excited strings. As each individual string carries a lot of internal energy (to produce the later secondaries) in a small and localized space-time volume the quarkonia state might get completely dissociated by the intense color electric field inside a single string 41 .

In the transport treatment one explicitely follow the motion of the $c \bar{c}$ pair in the (pre-)hadronic matter throughout the collision dynamics. The $c \bar{c}$ pair may now be either destroyed in collisions with pucleons with a dissociation crosssection of $3-6 \mathrm{mb}$ (see the discussion ind) or by dissociation on the very energetic prehadronic excitations. Several hundred of these strings are temporarily formed during a central $\mathrm{Pb}-\mathrm{Pb}$ collision at SPS energies in the early collision stage. The dynamical evolution of the strings is now included explicitly 6 . The fragmentation of the strings into hadrons starts after some phenomenologically accepted formation time $\tau_{f} \approx 0.8 \mathrm{fm} / \mathrm{c}$. For the

luedcg: submitted to World Scientific on October 25, 2018 

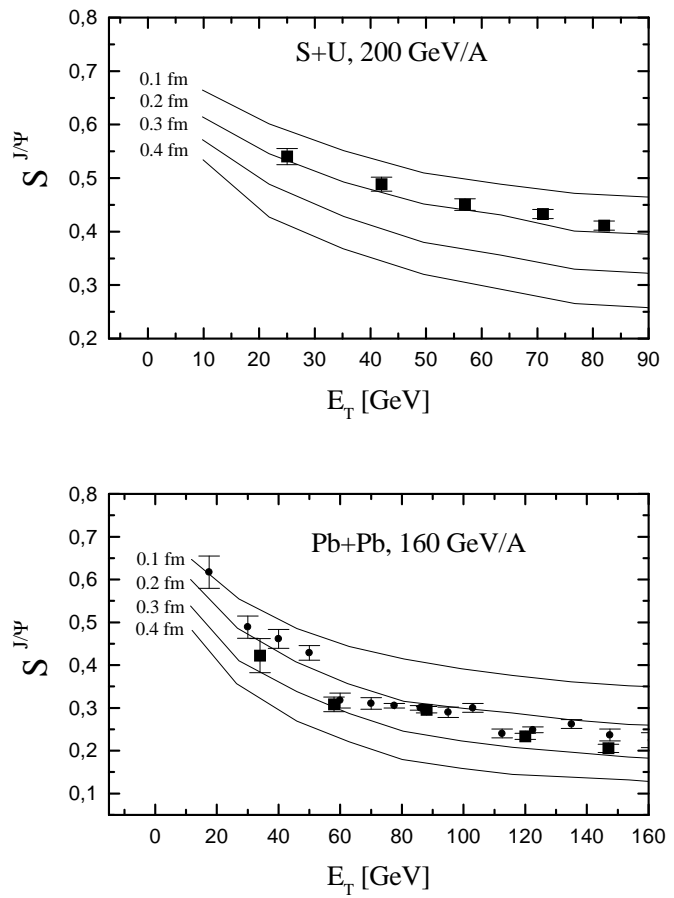

Figure 3. The $J / \Psi$ survival probability $S^{J / \Psi}$ for $\mathrm{S}+\mathrm{U}$ at $200 \mathrm{~A} \cdot \mathrm{GeV}$ (upper part) and $\mathrm{Pb}+\mathrm{Pb}$ at $160 \mathrm{~A} \cdot \mathrm{GeV}$ (lower part) as a function of the transverse energy $E_{T}$ in comparison to experimental data (full squares and circles). The calculated results are shown for the string radii $R_{s}=0.1,0.2,0.3$ and $0.4 \mathrm{fm}$.

dissociation we assume further that a $c \bar{c}$ state immediately gets broken apart whenever it moves into the region of the color electric field of a string. In this sense strings are completely 'black' for $c \bar{c}$ states 1 . The field energy density contained in a string is given by $\sigma /\left(\pi R_{S}^{2}\right)$, where $\sigma \approx 1 \mathrm{GeV} / \mathrm{fm}$ denotes the QCD string tension. For a string radius $R_{S} \approx 0.3 \mathrm{fm}$ one accordingly has a local high color electric energy density of $\approx 4 \mathrm{GeV} / \mathrm{fm} \mathrm{m}^{3}$, which substantially screens the binding potential of the charmonium state 3 .

The comparison to $J / \Psi$ suppression in nucleus-nucleus collisions is now performed on an event-by-event basis using the neutral transverse energy $E_{T}$ as a trigger as in the experiments. For $\mathrm{p}+\mathrm{U}$ and a string radius of $R_{S}=0.4$ fm only $2 \%$ of the $J / \Psi$ 's are absorbed by strings. The absorption is thus dominated, as expected intuitively, by the $c \bar{c}$-baryon dissociation on nucleons. 
This turns out to be completely different for heavy-ion collisions, where the absorption on strings becomes a much more important effect. In Fig. 3 our results are shown for $\mathrm{S}+\mathrm{U}$ and $\mathrm{Pb}+\mathrm{Pb}$ as a function of the transverse energy and for different string radii $R_{s}=0.1, \ldots, 0.4 \mathrm{fm}$. The later serves as the one phenomenological parameter to be adressed. A moderate to strong dependence on the string radius $R_{s}$ is observed with $R_{s} \approx 0.2-0.3 \mathrm{fm}$ giving the best fit to the experimental data. For this string radius $40 \%$ of the absorbed $J / \Psi$ 's are dissociated by strings in central collisions of $\mathrm{Pb}+\mathrm{Pb}$.

To summarize, various (hadronic) models can at present achieve a rather good agreement to all data. The underlying ideas are to some extent different. It is, of course, also possible that all of them might attribute to the suppression of $c \bar{c}$ states, so that the obliged requirement of a QGP to understand the results of most central $\mathrm{Pb}+\mathrm{Pb}$ collisions is not really given.

\section{Stochastic Disoriented Chiral Condensates}

In this last section we will give a brief report on our recent findings on the stochastic nature of DCC formation and how to possibly identify their existence experimentally $\mathrm{B}$. This work resulted from an earlier investigation 2 , where we adressed for the first time the potential likeliness of an instability leading to a sufficiently large DCC event during the evolution of a fireball undergoing a phase transition within the linear $\sigma$-model.

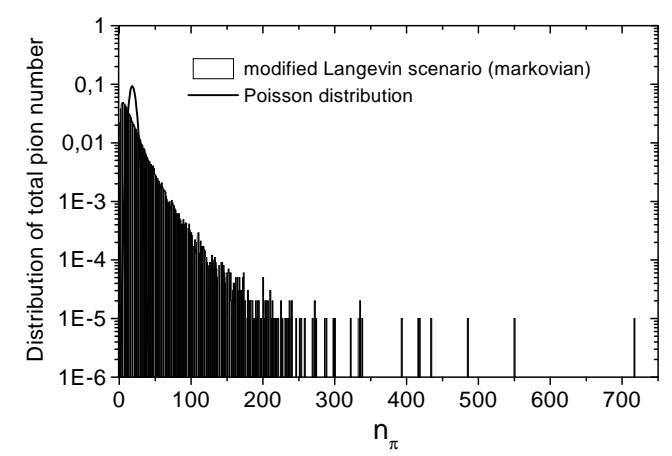

Figure 4. Statistical distribution $P\left(n_{\pi}\right)$ of the final yield $n_{\pi}$ in low momentum pion number of a single DCC for a rapidly expanding situation (see ref 8 for details) compared with a corresponding simple poissonian distribution. 
The main idea is that the final fluctuations depend critically on the initial conditions chosen for the evolving chiral order parameter, thus deciding to some extent whether the system enters temporarity the instable region during the 'roll-down' period of the order parameter120. In fact, a semi-classical and dissipative dynamics of the order parameter and the pionic fields can be obtained by an effective and complex action, where the interaction with the thermal pions has been integrated out. (One of the most prominent topics in modern statistical quantum field theory is to describe the evolution and behavior of the long wavelength modes at or near thermal equilibrium and also to understand the non-equilibrium evolution of a phase transition.) To the end, we have utilized the following stochastic Langevin equations of motion for the order parameters $\Phi_{a}=\frac{1}{V} \int d^{3} x \phi_{a}(\mathbf{x}, t)$ in a D-dimensional ('Hubble') expanding volume $V(\tau)$ to describe the evolution of collective pion and sigma fields 12, :

$$
\begin{aligned}
& \ddot{\Phi}_{0}+\left(\frac{D}{\tau}+\eta\right) \dot{\Phi}_{0}+m_{T}^{2} \Phi_{0}=f_{\pi} m_{\pi}^{2}+\xi_{0}, \\
& \ddot{\Phi}_{i}+\left(\frac{D}{\tau}+\eta\right) \dot{\Phi}_{i}+m_{T}^{2} \Phi_{i}=\xi_{i},
\end{aligned}
$$

with $\Phi_{0}=\sigma$ and $\Phi_{i}=\left(\pi_{1}, \pi_{2}, \pi_{3}\right)$ being the chiral meson fields and $m_{T}^{2}=\lambda\left(\Phi_{0}^{2}+\sum_{i} \Phi_{i}^{2}+\frac{1}{2} T^{2}-f_{\pi}^{2}\right)+m_{\pi}^{2}$ denotes the effective transversal ('pionic') masses. These coupled Langevin equations resemble in its structure a phenomenological Ginzburg-Landau description of phase transition. Aside from a theoretical justification one can regard the Langevin equation as a practical tool to study the effect of thermalization on a subsystem, to sample a large set of possible trajectories in the evolution, and to address also the question of all thermodynamically possible initial configurations in a systematic manner.

In fig. A we show the statistical distribution in the number of produced long wavelength pions $N_{\pi}$ out of the evolving chiral order fields within the DCC domain $V(\tau)$ for one particular set of parameters. A rather rapid and $(D=) 3$-dimensional expansion has been employed. (The results majorly depend on how fast the assumed cooling and expansion proceeds.) In general one finds that only for $\mathrm{D}=3$ and sufficiently fast expansion individual unusual strong fluctuations of the order of $50-200$ pions might occur, although the average number $\left\langle n_{\pi}\right\rangle$ of the emerging long wavelength pions only posesses a moderate and undetectable value of $5-20$.

In these interesting cases the final distribution does not follow a usual Poissonian distribution (comp. fig. (1), which represents a very important outcome of our investigation. (Critical, dynamical) Fluctuations with a large 


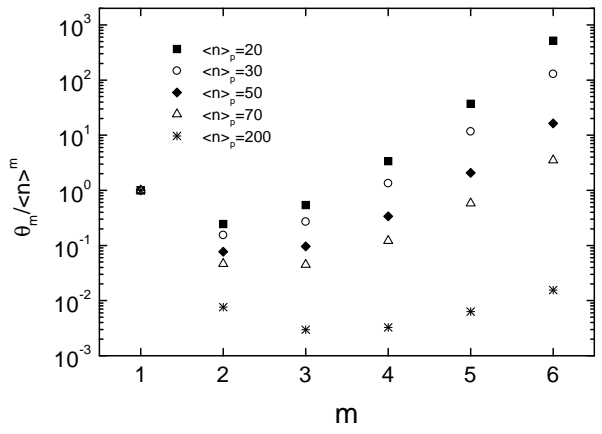

Figure 5. The reduced factorial cumulants for $m=1$ to 6 for the pion number distribution of low momentum stemming from a single emerging DCC (of the previous figure) and an additional poissonian distributed background pion source with different mean values $\langle n\rangle_{P}=20-200$.

number of produced pions are still likely with some small but finite probability! Unusual events out of sample contain a multiple in the number of pions compared to the average. One should indeed interpret those particular events as semi-classical 'pion bursts' similar to the mystique Centauro candidates. This result suggests a very important conclusion: If DCCs are being produced, an experimental finding will be a rare event following a strikingly, nontrivial and nonpoissonian distribution. A dedicated event-by-event analysis for the experimental programs (e.g. the STAR TPC at RHIC) is then unalterable.

The further analysis of this unusual distribution by means of the cumulant expansion shows that the reduced higher order factorial cumulants $\theta_{m} /<n_{\pi}>^{m}$ for $m \geq 3$ exhibit an abnormal, exponentially increasing tendency, as illustrated in fig. . There an additional incoherent Poissonian background of (low momentum) pions stemming from other possible sources has been added. We advocate that an analysis by means of the higher order cumulants serves as a new and powerful signature. In conclusion, the occurence of a rapid chiral phase transition (and thus DCCs) might then probably only be identified experimentally by inspecting higher order facorial cumulants $\theta_{m}$ $(m \geq 3)$ for taken distributions of low momentum pions. 


\section{Acknowledgments}

The topics reviewed have been done in various collaborations with T. Biro, E. Bratkovskaya, W. Cassing, J. Geiss, S. Leupold, S. Loh, U. Mosel and Z. Xu. This work has been supported by BMBF, DFG and GSI Darmstadt.

\section{References}

1. U. Heinz and M. Jacob, 'Evidence for a New State of Matter: An Assessment of the Result from the CERN Lead Beam Programme', CERN Press Office (2000), nucl-th/0002042.

2. P. Koch, B. Müller and J. Rafelski, Phys. Rep. 142, 167 (1986).

3. T. Matsui and H. Satz, Phys. Lett. B 178, 416 (1986).

4. J. Cleymans, contribution to this conference.

5. C. Greiner and S. Leupold, nucl-th/0009036; C. Greiner, nuclth/0011026.

6. J. Geiss, C. Greiner, E. Bratkovskaya, W. Cassing and U. Mosel, Phys. Lett. B 447, 31 (1999).

7. D. Anselm, Phys. Lett. B 217, 169 (1989); J.D. Bjorken, Int. J. Mod. Phys. A 7, 4819 (1992); K. Rajagopal and F. Wilczek, Nucl. Phys. B 404, 577 (1993).

8. Z. Xu and C. Greiner, Phys. Rev. D 62, 036012 (2000).

9. J. Geiss, W. Cassing and C. Greiner, Nucl. Phys. A 644, 107 (1998).

10. J. Geiss, PHD thesis, Universität Giessen (1998); W. Cassing, Nucl. Phys. A 661, 468c (1999).

11. S. Loh, C. Greiner and U. Mosel, Phys. Lett. B 404, 238 (1997).

12. T.S. Biró and C. Greiner, Phys. Rev. Lett. 79, 3138 (1997). 